Geometry $\&$ Topology

Volume 9 (2005) 179-202

Published: 8 January 2005

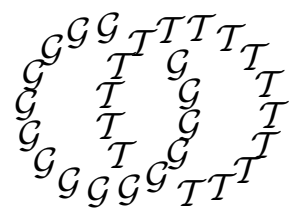

\title{
A characterization of short curves of a Teichmüller geodesic
}

\author{
KASRA RAFI \\ Department of Mathematics, University of Connecticut \\ Storrs, CT 06269, USA \\ Email: rafi@math.uconn.edu
}

\begin{abstract}
We provide a combinatorial condition characterizing curves that are short along a Teichmüller geodesic. This condition is closely related to the condition provided by Minsky for curves in a hyperbolic 3-manifold to be short. We show that short curves in a hyperbolic manifold homeomorphic to $S \times \mathbb{R}$ are also short in the corresponding Teichmüller geodesic, and we provide examples demonstrating that the converse is not true.
\end{abstract}

\section{AMS Classification numbers Primary: 30F60}

Secondary: $\quad 32 \mathrm{G} 15,30 \mathrm{~F} 40,57 \mathrm{M} 07$

Keywords: Teichmüller space, geodesic, short curves, complex of curves, Kleinian group, bounded geometry

Proposed: Benson Farb

Seconded: Jean-Pierre Otal, Walter Neumann
Received: 11 May 2004 Accepted: 27 December 2004 


\section{Introduction}

We are interested in studying the behavior of geodesics in Teichmüller space. Let $S$ be a surface of finite type, $\mathcal{T}(S)$ be the Teichmüller space of $S$ and $\hat{\mathcal{T}}(S)$ be the Thurston compactification of $\mathcal{T}(S)$. The boundary of $\hat{\mathcal{T}}(S)$ is the space of projectivized measure foliations, $\mathcal{P} \mathcal{M F}(S)$. A geodesic $g$ in $\mathcal{T}(S)$ can be described by a one-parameter family of quadratic differentials $q_{t}$ on $S$. The horizontal and the vertical foliations of $q_{t}$ define two elements, $\mu^{+}$and $\mu^{-}$, in $\mathcal{P} \mathcal{M F}(S)$ which are independent of $t$. We give a characterization of short curves of $\left(g, \mu^{+}, \mu^{-}\right)$based on the topological type of $\mu^{+}$and $\mu^{-}$only. Define

$$
l_{g}(\alpha)=\inf _{\sigma \in g} l_{\sigma}(\alpha)
$$

to be the shortest hyperbolic length of $\alpha$ over all hyperbolic metrics along the geodesic $g$. For a fixed $\epsilon>0$, we say $\alpha$ is short along $g$ if $l_{g}(\alpha) \leq \epsilon$. Also, for a fixed $K>0$ we say two essential arcs in a surface are quasi-parallel if their intersection number is less than $K$. The following is our main theorem:

Theorem 1.1 Let $\left(g, \mu^{+}, \mu^{-}\right)$be a geodesic in $\mathcal{T}(S)$ and $\alpha$ be a simple closed curve in $S$. Then $\alpha$ is short along $g$ if and only if, for some component $Y$ of $X \backslash \alpha$, the restrictions of $\mu^{+}$and $\mu^{-}$to $Y$ have no common quasi-parallels 1

Corollary 1.2 The set of short curves in $\left(g, \mu^{+}, \mu^{-}\right)$is independent of the measure class given on $\mu^{+}$and $\mu^{-}$.

This corollary sheds some light on the behavior of geodesics whose "endpoints" are not uniquely ergodic. We will discuss this and other applications of Theorem 1.1 in studying the geometry of $\mathcal{T}(S)$ and its stable and unstable geodesics in future papers.

The proof of the main theorem requires close attention to the geometry of the singular Euclidean metric defined on $S$ by a quadratic differential. An essential step is to prove an analogue of the collar lemma, which compares the lengths, in quadratic differential metric, of intersecting curves, assuming one of them has bounded hyperbolic length. Let $X$ be a Riemann surface, $q$ be a quadratic differential on $X$ and $\sigma$ be the corresponding hyperbolic metric on $X$.

Theorem 1.3 For every $L>0$, there exists $D_{L}$ such that, if $\alpha$ and $\beta$ are two simple closed curves in $X$ intersecting nontrivially with $l_{\sigma}(\beta) \leq L$, then

$$
D_{L} l_{q}(\alpha) \geq l_{q}(\beta) \text {. }
$$

\footnotetext{
${ }^{1}$ See Theorem 6.1 and Remark 6.2
} 
Relation with Kleinian groups The original motivation for this work was the question of whether the universal curve over a Teichmüller geodesic can be used to model an infinite-volume hyperbolic 3-manifold. Here we briefly discuss the connection between geodesics in $\mathcal{T}(S)$ and hyperbolic 3-manifolds and present some difficulties one might encounter in attempting to make such a model. In [16, Rees recently proposed a solution to these difficulties.

Let $M=S \times[0,1]$, and let $\mathbf{h}(M)$ be the space of complete hyperbolic structures on the interior of $M$. Elements of $\mathbf{h}(M)$ are infinite-volume hyperbolic 3manifolds homeomorphic to $S \times \mathbb{R}$. The intersection of $N \in \mathbf{h}(M)$ with a neighborhood of a boundary component of $M$ is called an end of $N$. With each end one can associate an invariant $\mu$ in an enlargement of $\mathcal{T}(S)$ related to Thurston's compactification of the Teichmüller space. If an end is geometrically finite, that is, if the conformal structure on $S \times\{t\}$ stabilizes as $t$ approaches infinity, $\mu$ is defined to be the limiting conformal structure on $S$, i.e., it is a point in $\mathcal{T}(S)$. If an end is degenerate, then $\mu$ is an "unmeasured" foliation on $S 2$ The ending lamination theorem states that these invariants are sufficient to determine the hyperbolic 3 -manifold $N$.

Theorem 1.4 (Brock, Canary, Minsky) The topological type of $N$ and its end invariants determine $N$ up to isometry.

The proof of the theorem involves building a model manifold for the hyperbolic 3-manifold $\left(N, \mu^{+}, \mu^{-}\right) \in \mathbf{h}(M)$ based on combinatorial information given by $\mu^{+}$and $\mu^{-}$(see 13]). In particular, short curves in $N$ are exactly the boundary components of surfaces $Y$ such that projections of $\mu^{+}$and $\mu^{-}$to $Y$ are far apart in the complex of curves of $Y$, that is, $d_{Y}\left(\mu^{+}, \mu^{-}\right)$is large (see [8] for definition and discussion). This condition is very closely related to the combinatorial condition given in Theorem 1.1. Consider the geodesic $\left(g, \mu^{+}, \mu^{-}\right)$in $\mathcal{T}(S)$. (We are abusing the notation here, since end invariants of $N$ are unmeasured foliations and $\mu^{+}$and $\mu^{-}$are in $\mathcal{P} \mathcal{M F}(S)$.) We say $N$ has bounded geometry if the injectivity radius of $N$ is positive, and we say $g$ has bounded geometry if, for some $\epsilon>0$, the injectivity radius of the hyperbolic metric at $g(t)$ is larger than $\epsilon$. Using the technology developed in [8] and [13] we prove:

Theorem 1.5 The following are equivalent:

(1) $N$ has bounded geometry,

\footnotetext{
${ }^{2}$ An end invariant, in general, is a "hybrid" of a conformal structure and an unmeasured foliation.
} 
(2) $g$ has bounded geometry 3

Theorem 1.6 For every $\epsilon$ there exists $\epsilon^{\prime}$ such that, if the length of $\alpha$ in $N$ is less than $\epsilon^{\prime}$, then $l_{g}(\alpha) \leq \epsilon$.

The converse of the last theorem is not true. In Section 7 we will provide a family of counterexamples.

Theorem 1.7 There exist a sequence of manifolds $\left(N_{n}, \mu_{n}^{+}, \mu_{n}^{-}\right)$, corresponding Teichmüller geodesics $\left(g_{n}, \mu_{n}^{+}, \mu_{n}^{-}\right)$and a curve $\gamma$ in $S$ such that $l_{N_{n}}(\gamma) \geq$ $c>0$ for all $n$, but

$$
l_{g_{n}}(\gamma) \rightarrow 0 \quad \text { as } \quad n \rightarrow \infty
$$

Outline of the paper We begin in Section 2 by introducing subsurface projections and reviewing some of their properties. In Section 3 we discuss the geometry of a quadratic differential metric on a Riemann surface and the behavior of geodesics there. In Section 4 we prove the "collar lemma" (Theorem 1.3). Theorems establishing a connection between the combinatorial properties of horizontal and vertical foliations and the short curves in the quadratic differential metric are presented in Section [5 The main theorem is proven in Section [6. and Theorems 1.5] 1.6 and 1.7 are proven in Section [7.

Notation To simplify our presentation we use the notations $O, \succ$ and $\asymp$, defined as follows: for two functions $f$ and $g, f \succ g$ means $f \geq c g-d, f \asymp g$ means $c f-d \leq g \leq C f+D$, and $f=O(g)$ means $f \leq C g$, where $c, d, C$ and $D$ depend on the topology of $S$ only.

Acknowledgment I would like to thank the referee for careful reading and for numerous comments and corrections.

\section{Simple closed curves}

In this section we study some properties of arcs and curves on surfaces. Let $S$ be an orientable surface of finite type, excluding the sphere and the torus. By a curve we mean a non-trivial, non-peripheral, piecewise smooth simple closed curve in $S$. The free homotopy class of a curve $\alpha$ is denoted by $[\alpha]$. By an essential arc $\omega$ we mean a piecewise smooth simple arc, with endpoints on the

\footnotetext{
${ }^{3}$ This theorem is also a corollary of Theorem 1.4 and [10.
} 
boundary of $S$, that cannot be pushed to the boundary of $S$. In case $S$ is not an annulus, $[\omega]$ represents the homotopy class of $\omega$ relative to the boundary of $S$. When $S$ is an annulus, $[\omega]$ is defined to be the homotopy class of $\omega$ relative to the endpoints of $\omega$.

Define $\mathcal{C}(S)$ to be the set of all homotopy classes of curves and essential arcs on the surface $S$. A curve system $\Gamma=\left\{\gamma_{1}, \ldots, \gamma_{n}\right\}$ is a non-empty set of curves and essential arcs in $S$ that are pairwise disjoint from each other. The homotopy class of $\Gamma$ is the set of homotopy classes of elements of $\Gamma,[\Gamma]=$ $\left\{\left[\gamma_{1}\right], \ldots,\left[\gamma_{n}\right]\right\}$. Let $\mathcal{C} S(S)$ be the space of all classes of curve systems on $S$. For two curve systems $\Gamma_{1}$ and $\Gamma_{2}$ we define the intersection number between $\Gamma_{1}$ and $\Gamma_{2}, i_{S}\left(\Gamma_{1}, \Gamma_{2}\right)$, to be the minimum geometric intersection number between an element of $\Gamma_{1}$ and an element of $\Gamma_{2}$. This depends only on the homotopy classes of $\Gamma_{1}$ and $\Gamma_{2}$. If $S$ is an annulus, $\Gamma_{1}$ includes the core curve of $S$ and $\Gamma_{2}$ includes any transverse curve, then we define their intersection number to be infinity.

A subsurface $Y$ of $S$ is a connected open subset of $S$ with piecewise smooth boundary. Let $[Y]$ denote the homotopy class of $Y$, that is, the set of all connected open subsets of $S$ with piecewise smooth boundary that are homotopically equivalent to $Y$. A choice of base point in $Y$ identifies $\pi_{1}(Y)$ with a subgroup of $\pi_{1}(S)$. In fact, this subgroup is well defined up to conjugation for $[Y]$.

Let $\mu$ be a curve system, a lamination or a singular-foliation on $S$. Here we define the projection of $\mu$ to the subsurface $Y$. Let

$$
f: \bar{S} \rightarrow S
$$

be a covering of $S$ such that $f_{*}\left(\pi_{1}(\bar{S})\right)$ is conjugate to $\pi_{1}(Y)$. We call this a $Y$-cover of $S$. Since $S$ admits a hyperbolic metric, $\bar{S}$ has a well defined boundary at infinity. Let $\bar{\mu}$ be the lift of $\mu$ to $\bar{S}$. Components of $\bar{\mu}$ that are curves or essential arcs on $\bar{S}$, if any, form a curve system in $\bar{S}$. This set, if not empty, defines an element in $\mathcal{C} S(\bar{S})$. Since $\bar{S}$ is homeomorphic to $Y, \mathcal{C} S(\bar{S})$ is identified with $\mathcal{C} S(Y)$ through this homeomorphism. We call the corresponding element of $\mathcal{C} S(Y)$ the projection of $\mu$ to $Y$ and will denote it by $\mu_{Y}$. If there are no essential arcs or curves in $\bar{\mu}, \mu_{Y}$ is the empty set; otherwise we say that $\mu$ intersects $Y$ non-trivially. Note that this projection depends on the homotopy class of $\mu$ only.

Subsurface intersection Let $\mu$ and $\nu$ be two curve systems, laminations or singular foliations on $S$ that intersect a subsurface $Y$ non-trivially. We define 
the $Y$-intersection of $\mu$ and $\nu$ to be the intersection number in $Y$ between the projections $\mu_{Y}$ and $\nu_{Y}$ and denote it by

$$
i_{Y}(\mu, \nu)=i_{Y}\left(\mu_{Y}, \nu_{Y}\right) .
$$

\section{Quadratic differentials}

In this chapter we review the geometry of quadratic differentials. For more detailed discussion, see [9] and [17.

Let $\hat{X}$ be a compact Riemann surface and let $q \not \equiv 0$ be a meromorphic quadratic differential on $\hat{X}$. We assume that the set of critical points of $q$ is discrete and that $q$ has finite critical points only (i.e., critical points of $q$ are either zeros or poles of order 1). The surface $\hat{X}$ punctured at poles $P_{i}$ of $q$ is denoted by $X$. For any such $q,|q|$ defines a singular Euclidean metric in $X$. We denote the length of a piecewise smooth curve by $l_{q}(\gamma)$ and the area of a subset $Y$ of $X$ by $\operatorname{area}_{q}(Y)$. We also assume that $\operatorname{area}_{q}(X)=1$. The metric defined on $X$ is not complete since the finite poles are finite distance from interior points of $X$. Let $\tilde{X}$ be the completion of the universal cover of $X$. In a neighborhood of every regular point $P$ of $q$ we can introduce a local parameter $w$, in terms of which the representation of $q$ is identically equal to one. This parameter, given by the integral

$$
w=Q(z)=\int \sqrt{q(z)} d z
$$

is uniquely determined up to a transformation $w \rightarrow \pm w+$ const, and it will be called the natural parameter near $P$.

Horizontal and vertical foliations A straight arc is a smooth curve $\gamma$ such that, for every point $P$ in $\gamma$, the image of $\gamma$ under the natural parameter near $P$ is a straight line. Since $w$ is "unique", the angle of $\gamma$ is a well-defined number $0 \leq \theta<\pi$. A maximal such straight arc is called a $\theta$-trajectory. A straight arc connecting two critical points in $(X, q)$ is called a straight segment. The $\theta$-trajectory passing through a regular point of $q$ is unique. A trajectory is called a critical trajectory if at least one of its ends tends to a critical point. There are only finitely many critical $\theta$-trajectories.

For each $\theta$, the $\theta$-trajectories foliate the set of regular points in $X$. For $n=$ $-1,1,2$, a local neighborhood of a finite critical point of degree $n$ is shown in Figure 1 


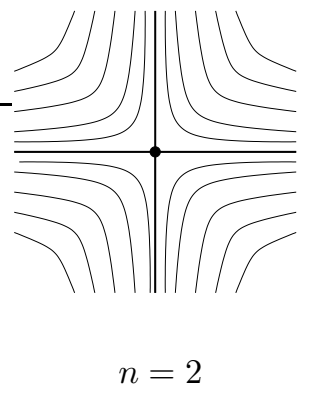

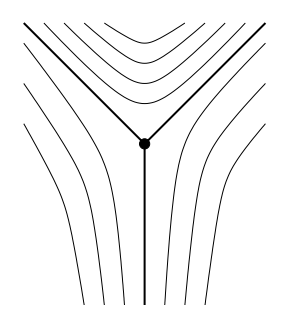

$n=1$

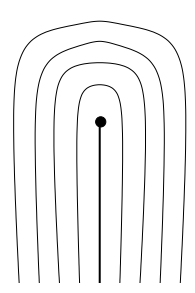

$n=-1$

Figure 1: Local pictures of a foliation by trajectories

The foliation corresponding to $\theta=0$ (respectively, $\theta=\pi / 2$ ), is called the horizontal foliation (the vertical foliation) and is denoted by $\nu_{+}\left(\nu_{-}\right)$. Let $\gamma$ be any closed curve or arc. Chop $\gamma$ to coordinate patch sized segments $\gamma_{i}$. For each such segment, we define the horizontal length to be

$$
h_{q}\left(\gamma_{i}\right)=\int_{0}^{1}\left|\sqrt{q\left(\gamma_{i}(t)\right)}\right| \cdot\left|\operatorname{Re}\left\{\gamma_{i}^{\prime}(t)\right\}\right| d t
$$

Then, the horizontal length of $\gamma, h_{q}(\gamma)$, is defined as the sum of horizontal lengths of the $\gamma_{i}$. The vertical length of $\gamma$ is defined similarly and is denoted by $v_{q}(\gamma)$. Let $\gamma$ be an arc that is transverse to the horizontal foliation. The vertical length of $\gamma$ does not change after a homotopic change through arcs that are transverse to the horizontal foliation. Therefore the horizontal length defines a transverse measure on $\nu_{-}$; similarly, the vertical length defines a transverse measure on $\nu_{+}$, making $\nu_{+}$and $\nu_{-}$into measured foliations.

Geodesic representatives We would like to define the $q$-geodesic representative of a curve $\gamma$ to be the curve in the free homotopy class of $\gamma$ with the shortest $q$-length. If $X$ has no punctures, the $q$-metric is complete and the geodesic representative always exists. In general, the $q$-metric on $X$ is not complete; however, for any lift $\tilde{\gamma}$ of $\gamma$, there exists a unique geodesic $\tilde{\gamma}_{q}$ in $\tilde{X}$ that has the same endpoints at infinity as $\tilde{\gamma}$. The projection of $\tilde{\gamma}_{q}$ to $\hat{X}$ is denoted by $\gamma_{q}$ and is called the $q$-geodesic representative of $\gamma$. The curve $\gamma_{q}$ is composed of straight segments which meet at critical points of $X$, making an angle of at least $\pi$ on either side. If $\gamma_{q}$ "passes through" a pole of $\hat{X}$, it makes at least a full turn around the pole.

Unfortunately, one cannot recover the homotopy class of $\gamma$ from the set $\gamma_{q}$. Therefore, we should think of $\gamma_{q}$ as a parametrized curve. Identify $\tilde{\gamma}_{q}$ with the real line as a metric space. The deck transformations fixing $\tilde{\gamma}_{q}$ define a $\mathbb{Z}$ 
action on it. The quotient is a circle of length $l_{q}(\gamma)$. The projection from $\tilde{X}$ to $\hat{X}$ defines a map

$$
f_{\gamma}: S^{1} \rightarrow \gamma_{q}
$$

which we call the natural parametrization of $\gamma_{q}$.

Theorem 3.1 (see [9], 17]) For $(X, q)$ as above,

(1) Let $\gamma$ be an arc joining two given points $x$ and $y$ of $X$; then the $q-$ geodesic representative of $\gamma$ exists and is unique.

(2) Let $\gamma$ be an essential loop on $X$; then the $q$-geodesic representative of $\gamma$ exists and is unique, except for the case where it is one of the continuous family of closed Euclidean geodesics in a flat annulus (see page 187 for definition of a flat annulus).

A geodesic curve or arc $\gamma$ is called balanced if $v_{q}(\gamma)=h_{q}(\gamma)$. If $v_{q}(\gamma) \geq h_{q}(\gamma)$ we say $\gamma$ is mostly vertical and if $v_{q}(\gamma) \leq h_{q}(\gamma)$ we say $\gamma$ is mostly horizontal. Consider $\gamma$ as a union of straight segments $\gamma_{i}$. Let $l_{i}, h_{i}$ and $v_{i}$ be the $q-$ length, the horizontal length and the vertical length of $\gamma_{i}$ respectively. We have $l_{i}^{2}=h_{i}^{2}+v_{i}^{2}$. The Cauchy-Schwarz inequality implies

$$
\begin{aligned}
l_{q}(\gamma)^{2} & =\left(\sum l_{i}\right)^{2} \leq 2 \sum l_{i}^{2}=2 \sum h_{i}^{2}+2 \sum v_{i}^{2} \\
& \leq 2\left(\sum h_{i}\right)^{2}+2\left(\sum v_{i}\right)^{2}=2\left(h_{q}(\gamma)^{2}+v_{q}(\gamma)^{2}\right) .
\end{aligned}
$$

Therefore, if $\gamma$ is mostly vertical (respectively, mostly horizontal), then

$$
2 v_{q}(\gamma) \geq l_{q}(\gamma) \quad\left(\text { respectively, } \quad 2 h_{q}(\gamma) \geq l_{q}(\gamma)\right) \text {. }
$$

Curvature of a curve in $X$ Let $\gamma$ be a boundary component of $Y$. The curvature of $\gamma$ with respect to $Y, \kappa_{Y}(\gamma)$, is well defined as a measure with atoms at the corners (that is, $(\pi-\theta)$, where $\theta$ is the interior angle at the corner). We choose the sign to be positive when the acceleration vector points into $Y$. If $\gamma$ is curved positively (or negatively) with respect to $Y$ at every point, we say it is monotonically curved with respect to $Y$.

Remark 3.2 The curvature of any closed curve is a multiple of $\pi$ (see [9]).

Theorem 3.3 (Gauss-Bonnet) Let $Y$ be a subsurface of $\hat{X}$ with boundary components $\gamma_{1}, \ldots, \gamma_{n}$; also let $P_{1}, \ldots, P_{k}$ be the critical points in $Y$. Then we have

$$
\sum_{i=1}^{n} \kappa_{Y}\left(\gamma_{i}\right)=2 \pi \chi(Y)+\pi\left(\sum_{i=1}^{k} \operatorname{deg}\left(P_{i}\right)\right),
$$

where $\operatorname{deg}\left(P_{i}\right)$ is the order of the critical point $P_{i}$. 
Regular and primitive annuli in $X$ In general, it is difficult to estimate the modulus of an annulus in $X$. Here we discuss estimates for moduli of certain kinds of annuli, which will be useful in estimating the hyperbolic length of a closed curve (see [9, Section 4]).

Definition 3.4 Let $A$ be an open annulus in $X$ with boundaries $\gamma_{0}$ and $\gamma_{1}$. Suppose both boundaries are monotonically curved with respect to $A$. Further, suppose that the boundaries are equidistant from each other, and $\kappa_{A}\left(\gamma_{0}\right) \leq 0$. We call $A$ a regular annulus. If $\kappa_{A}\left(\gamma_{0}\right)<0$, we call $A$ expanding and say that $\gamma_{0}$ is the inner boundary and $\gamma_{1}$ is the outer boundary. If the interior of $A$ contains no zeroes, we say $A$ is a primitive annulus, and we write $\kappa(A)=-\kappa_{A}\left(\gamma_{0}\right)$. When $\kappa(A)=0, A$ is a flat annulus and is foliated by closed Euclidean geodesics homotopic to the boundaries.

Remark 3.5 Later in this section we will discuss certain covers of $X$ which are not compact. There, a regular annulus can be infinite, having only one boundary component, the inner boundary.

Lemma 3.6 Let $A$ be a primitive annulus, $\gamma_{0}$ and $\gamma_{1}$ be the inner and the outer boundary of $A$ respectively and $d$ be the distance between the boundaries of $A$. Then

$$
\begin{cases}\kappa \operatorname{Mod}(A) \asymp \log \left(\frac{d}{l_{q}\left(\gamma_{0}\right)}\right) & \text { if } \kappa(A)>0, \\ \operatorname{Mod}(A) l_{q}\left(\gamma_{0}\right)=d\left(\gamma_{0}, \gamma_{1}\right) & \text { if } \kappa(A)=0 .\end{cases}
$$

Proof The statement is clear for a flat annulus. In case $\kappa(A)>0$, Minsky has shown ([9, Theorem 4.5]) that

$$
\operatorname{Mod}(A) \asymp \frac{1}{\kappa(A)} \log \left(\frac{l_{q}\left(\gamma_{1}\right)}{l_{q}\left(\gamma_{0}\right)}\right) .
$$

We have to show that $l_{q}\left(\gamma_{1}\right) / l_{q}\left(\gamma_{0}\right) \asymp d / l_{q}\left(\gamma_{0}\right)$. For $0 \leq t \leq d$, define $\alpha_{t}$ to be the equidistant curve from $\alpha_{0}$ with $d\left(\alpha_{0}, \alpha_{t}\right)=t$. Let $A_{t}$ be the annulus bounded by $\alpha_{0}$ and $\alpha_{t}$. The Gauss-Bonnet theorem implies that $\kappa_{A_{t}}\left(\alpha_{t}\right)+$ $\kappa_{A_{t}}\left(\alpha_{0}\right)=0$. Therefore, $\kappa_{A_{t}}\left(\alpha_{t}\right)=\kappa$. The $q$-length of $\alpha_{t}$ is a differentiable function of $t$ almost everywhere, and

$$
\frac{d}{d t} l_{q}\left(\alpha_{t}\right)=\kappa_{A_{t}}\left(\alpha_{t}\right)
$$

that is, $l_{q}\left(\alpha_{t}\right)=l_{q}\left(\alpha_{0}\right)+\kappa t$. Therefore, $l_{q}\left(\gamma_{1}\right)=l_{q}\left(\gamma_{0}\right)+\kappa d$. This finishes the proof. 
We also recall the following theorem of Minsky. This is a consequence of Theorem 4.6 and Theorem 4.5 in 9 . (The inequality $\operatorname{Mod}(A) \leq m_{0}$ in 9 , Theorem 4.6] is a typo and should $\operatorname{read} \operatorname{Mod}(A) \geq m_{0}$.)

Theorem 3.7 (Minsky 9]) If $A \subset X$ is any homotopically nontrivial annulus, then $A$ contains a primitive annulus $B$ such that

$$
\operatorname{Mod}(B) \asymp \operatorname{Mod}(A) .
$$

Subsurfaces of $X$ Given a subsurface $Y$, it is desirable to consider a representative of homotopy class $Y$ with $q$-geodesic boundary. The naïve approach (which would work for the hyperbolic metric) would be to pick a geodesic representative for each boundary component of $Y$ and take the complementary component of these curves in $X$ that is in the homotopy class of $Y$. However, the geodesic representatives of boundary components of $Y$ might be "tangent" to each other, that is, they might share a common geodesic segment. Also, a geodesic representative of a simple closed curve could have self-tangencies. As a result, it is possible that none of the complementary components are in the homotopy class of $Y$.

Here we describe a representative of $Y$ in the $Y$-cover of $X$ which, as we shall see, will be more convenient to work with. Let $\bar{X}$ be the $Y$-cover of $X$; for each boundary component $\alpha$ of $Y$, let $\bar{X}_{\alpha}$ be the annular cover of $X$ corresponding to $\alpha$; and let

$$
p: \bar{X} \rightarrow X, \quad \text { and } \quad p_{\alpha}: \bar{X}_{\alpha} \rightarrow \bar{X}
$$

be the corresponding covering maps. Consider the lift $\hat{Y}$ of $Y$ to $\bar{X}$ such that $p: \hat{Y} \rightarrow Y$ is a homeomorphism. The complement of $\hat{Y}$ in $\bar{X}$ is a union of annuli. We denote the annulus corresponding to a boundary component $\alpha$ by $B_{\alpha}$.

The geodesic representative of the core curve of $\bar{X}_{\alpha}$ is an embedded two-sided curve dividing $\bar{X}_{\alpha}$ into two annuli. The lift of $B_{\alpha}$ to $\bar{X}_{\alpha}$ shares an end with one of these annuli, which we denote by $\bar{A}_{\alpha}$. The interior of $\bar{A}_{\alpha}$ is mapped homeomorphically into $\bar{X}$ by $p_{\alpha}$. Otherwise, there would be a disk in $\bar{X}$ whose boundary consists of two geodesic arcs. This is a contradiction, because the geodesic representation of an arc is unique. Using the same argument we also have that the annuli corresponding to different boundary components of $\hat{Y}$ are disjoint. We denote $p_{\alpha}\left(\bar{A}_{\alpha}\right)$ by $A_{\alpha}$.

If $Y$ is not an annulus we define $\bar{Y}$ to be the complement in $\bar{X}$ of all such annuli $A_{\alpha}$. Otherwise let $\bar{Y}$ be the flat annulus containing all geodesic representatives 
of $\alpha$. The surface $\bar{Y}$ is closed and connected, and the restriction of $p$ to the interior of $\bar{Y}$ is one-to-one. Curves intersecting $Y$ essentially lift to arcs that have a compact restriction to $\bar{Y}$ and wander off to infinity from both ends into expanding annuli without ever coming back. That allows us to talk about the restriction of a curve to $\bar{Y}$ while keeping track of the homotopy type of the intersecting curve in the degenerate cases, where boundaries of $\bar{Y}$ are tangent to each other. As a consequence of the Gauss-Bonnet theorem in this setting we have:

Lemma 3.8 For two infinite geodesic arcs $\mu$ and $\nu$ in $\bar{X}$, the geometric intersection number between $\mu$ and $\nu$ is equal to the intersection number of the restrictions of $\mu$ and $\nu$ to $\bar{Y}$ up to an additive constant (in fact, the difference is at most 2).

\section{Collar lemma for a quadratic differential metric on a surface}

Let $X$ and $q$ be as before and $\sigma$ be the corresponding hyperbolic metric on $X$. In this section we prove Theorem 1.3

Theorem 1.3 For every $L>0$, there exists $D_{L}$ such that if $\alpha$ and $\beta$ are two simple closed curves in $X$ intersecting non-trivially with $l_{\sigma}(\beta) \leq L$, then

$$
D_{L} l_{q}(\alpha) \geq l_{q}(\beta) .
$$

Let $B_{\beta}$ be the $\delta$-neighborhood of the geodesic representative of $\beta$ in the hyperbolic metric of $X$, where $\delta$ is a largest number such that this neighborhood is an annulus, and $m$ be the modulus of $B_{\beta}$. We have (see [6])

$$
\log (m) \succ-L .
$$

Also let $\epsilon$ be such that $B_{\beta}$ is disjoint from the $\epsilon$-thin part of the hyperbolic metric on $X$. We can choose $\epsilon$ such that $\epsilon<L$ and $e^{L} \asymp \frac{1}{\epsilon}$. (The reader should keep in mind that the theorem is of interest for $L$ large. The condition $\epsilon<L$ is included here simply to ensure that the proof works for small values of $L$ as well.) Now pick $M \asymp \frac{1}{\epsilon}$ such that, if $A$ is an annulus with modulus greater than or equal to $M$, then the length of a core curve in $A$ in the complete hyperbolic metric on $A$ is less than $\epsilon$. The Schwarz lemma implies that the $\sigma$-length of this curve is also less than $\epsilon$ and therefore $B_{\beta}$ does not intersect the core of $A$. We need the following technical lemma. 
Lemma 4.1 Let $\beta$ and $\alpha$ be as above. Then there exists a subsurface $Z$ containing $B_{\beta}$ such that

$$
\operatorname{area}_{q}(Z) \leq R_{L} l_{q}\left(\alpha_{q}\right)^{2}
$$

where $R_{L}$ is a constant depending on $L$ and the topology of $X$.

Proof The strategy is to take $Z$ to be the smallest neighborhood of $\alpha_{q}$ that contains a primitive annulus of modulus $M$ in a neighborhood of each of its boundary components. Define $N_{r}$ to be the open $r$-neighborhood of $\alpha_{q}$ in $X$, and let $Z_{r}$ be the union of $N_{r}$ with all the components of $X-N_{r}$ that are disks or punctured disks. Let $K=-2 \pi \chi(X)$ and $\kappa_{r}$ be the sum of the curvatures of the boundary components of $Z_{r}$ with respect to $Z_{r}$. The Gauss-Bonnet theorem implies $4 \kappa_{r} \leq K$.

Observe that $l_{q}\left(\partial Z_{r}\right)$ and $\operatorname{area}_{q}\left(Z_{r}\right)$ are differentiable functions of $r$ almost everywhere and are continuous except at finitely many points, where we add a disk or a punctured disk. For differentiable points we have $\frac{d}{d r} l_{q}\left(\partial Z_{r}\right)=\kappa_{r}$. At any $r_{u}$ where we add a disk or a punctured disk $D_{u}$, the value of $l_{q}\left(\partial Z_{r}\right)$ decreases by the $q$-length of the boundary of $D_{u}$, which we call $c_{u}$. Let $I_{r}$ be the set of all indexes $u$ such that $r_{u} \leq r$. We have the following equation:

$$
l_{q}\left(\partial Z_{r}\right)-l_{q}\left(\partial Z_{0}\right)=\int_{0}^{r} \kappa_{\rho} d \rho-\sum_{u \in I_{r}} c_{u} .
$$

But $l_{q}\left(\partial Z_{0}\right)=2 l_{q}\left(\alpha_{q}\right)$ and $\kappa_{\rho} \leq K$. Therefore,

$$
l_{q}\left(\partial Z_{r}\right) \leq K r+2 l_{q}\left(\alpha_{q}\right)
$$

and

$$
\sum_{u \in I_{r}} c_{u} \leq K r+2 l_{q}\left(\alpha_{q}\right) .
$$

To find an upper bound for $\operatorname{area}_{q}\left(Z_{r}\right)$, we observe that

$$
\frac{d}{d r} \operatorname{area}_{q}\left(Z_{r}\right)=l_{q}\left(\partial Z_{r}\right) \text {. }
$$

At any $r_{u}$ where we add a disk or a punctured disk, $\operatorname{area}_{q}\left(Z_{r}\right)$ increases by $O\left(c_{u}^{2}\right) 5$ Therefore,

$$
\operatorname{area}_{q}\left(Z_{r}\right)-\operatorname{area}_{q}\left(Z_{0}\right)=\int_{0}^{r} l_{q}\left(\partial Z_{\rho}\right) d \rho+\sum_{u \in I_{r}} O\left(c_{u}^{2}\right) .
$$

\footnotetext{
${ }^{4}$ Use $\chi\left(Z_{r}\right) \leq 0$ and $\sum_{i=1}^{k} \operatorname{deg}\left(P_{i}\right) \leq-2 \chi(X)$.

${ }^{5}$ This is the isoperimetric inequality. For the punctured disk, where there is a point of positive curvature, consider the double-cover and add the missing point.
} 
But $\operatorname{area}_{q}\left(Z_{0}\right)=0$. Equation (4) and the Cauchy-Schwarz inequality imply

$$
\operatorname{area}_{q}\left(Z_{r}\right) \leq \int_{0}^{r}\left(K \rho+2 l_{q}\left(\alpha_{q}\right)\right) d \rho+\left[\sum_{u \in I_{r}} O\left(c_{u}\right)\right]^{2} .
$$

Now use (15) and take the integral to obtain

$$
\operatorname{area}_{q}\left(Z_{r}\right) \leq \frac{K r^{2}}{2}+2 l_{q}\left(\alpha_{q}\right) r+O\left(\left(K r+2 l_{q}\left(\alpha_{q}\right)\right)^{2}\right) .
$$

Let $r_{0}=0$ and $0<r_{1}<\ldots<r_{s}$ be points where the topology of $Z_{r}$ changes or a critical point of $X$ is added to $Z_{r}$. The set $Z_{r_{t+1}} \backslash Z_{r_{t}}$ is a union of primitive annuli. Let $t$ be the smallest index such that the moduli of all these primitive annuli are greater than $M$. If this never happens, let $t=s$.

For $j<t$ there exists a primitive annulus $B$ with modulus less than $M$ connecting a boundary component of $Z_{r_{j+1}}$ to a boundary component of $Z_{r_{j}}$. The distance between the boundaries of $B$ is $r_{j+1}-r_{j}$ and the length of the shorter boundary component of $B$ is at most $K r_{j}+2 l_{q}\left(\alpha_{q}\right)$ (Equation (4)). Using Lemma 3.6] there exists a $c>0$ so that

$$
r_{j+1}-r_{j} \leq c^{K M}\left(K r_{j}+2 l_{q}\left(\alpha_{q}\right)\right) .
$$

Let $P=c^{K M} K+1$ and $Q=2 c^{K M}$. We have

$$
r_{j+1} \leq P r_{j}+Q l_{q}\left(\alpha_{q}\right) .
$$

Now pick $\hat{r}>r_{t}$ to be the smallest number such that modulus of every annulus in $Z_{\hat{r}} \backslash Z_{r_{t}}$ is larger than or equal to $M$. This is possible by the choice of $t$. If $Z_{r_{t}}$ equals $X$, then $\hat{r}=r_{t}$, otherwise, by choosing $\hat{r}$ to be the smallest such number, we guarantee the existence of a primitive annulus of modulus exactly $M$. Then the above argument shows

$$
\hat{r} \leq P r_{t}+Q l_{q}\left(\alpha_{q}\right) .
$$

Combining (77) for $0 \leq j<t$ and (8) we get

$$
\hat{r} \leq P^{t+1} r_{0}+\sum_{i=0}^{t} P^{i} Q l_{q}\left(\alpha_{q}\right) .
$$

But $r_{0}=0$. Therefore

$$
\hat{r} \leq \frac{\left(P^{t+1}-1\right) Q}{P-1} l_{q}\left(\alpha_{q}\right) .
$$

Let $Z=Z_{\hat{r}}$. Combining this inequality with (6) we have, for an appropriate $R_{L}$,

$$
\operatorname{area}_{q}(Z) \leq R_{L} l_{q}\left(\alpha_{q}\right)^{2}
$$


Note that $R_{L}$ depends on $L$ or the topology of $X$ only. We have to show that $Z$ contains $B_{\beta}$. Let $A_{1}, \ldots, A_{k}$ be the annuli in $Z_{\hat{r}} \backslash Z_{r_{t}}$. Since $\beta$ intersects $\alpha$ essentially, $B_{\beta}$ has to intersect $Z$ essentially. But the modulus of any $A_{i}$ is larger than $M$; therefore $B_{\beta}$ is disjoint from a core curve in each $A_{i}$. That is, $B_{\beta}$ is contained in $Z$.

Proof of Theorem 1.3 Let $\lambda$ be the length of a shortest representative of curve $[\beta]$ in $B_{\beta}$ equipped with the $q$-metric inherited from $X$. By definition of modulus we have:

$$
\frac{\lambda^{2}}{\operatorname{area}_{q}\left(B_{\beta}\right)} \leq \frac{1}{m}
$$

But $l_{q}\left(\beta_{q}\right) \leq \lambda$ and $\operatorname{area}_{q}\left(B_{\beta}\right) \leq \operatorname{area}_{q}(Z)$. Therefore,

$$
\frac{l_{q}^{2}\left(\beta_{q}\right)}{\operatorname{area}_{q}(Z)} \leq \frac{\lambda^{2}}{\operatorname{area}_{q}\left(B_{\beta}\right)} .
$$

That is,

$$
l_{q}^{2}\left(\beta_{q}\right) \leq \frac{\operatorname{area}_{q}(Z)}{m} \leq \frac{R_{L} l_{q}^{2}\left(\alpha_{q}\right)}{m} .
$$

Setting $D_{L}=\sqrt{\frac{R_{L}}{m}}$ finishes the proof of the theorem.

Remark 4.2 Note that $\log \left(R_{L}\right) \asymp M \asymp e^{L}$ and $\log (m) \asymp-L$. Therefore

$$
\log \left(D_{L}\right) \asymp e^{L}+L \asymp e^{L} .
$$

Remark 4.3 The above estimate for $D_{L}$ is sharp. It is easy to produce examples where

$$
\frac{l_{q}(\beta)}{l_{q}(\alpha)} \geq e^{e^{L}}
$$

\section{Short curves of a quadratic differential metric}

Let $X, q, \nu_{+}$and $\nu_{-}$be as before, $Y$ be a subsurface of $X$ and $\bar{X}$, and $\bar{Y}$ be as in Section 3 Let $\bar{q}, \bar{\nu}_{+}$and $\bar{\nu}_{-}$be lifts of $q, \nu_{+}$and $\nu_{-}$to $\bar{X}$, respectively. Fix a positive constant $\delta$.

Definition 5.1 Let $f:[0,1] \times(0,1) \rightarrow \bar{X}$ be an embedding with the following properties:

(1) For $t \in(0,1), f$ maps $[0,1] \times\{t\}$ into a horizontal leaf of $q$. 
(2) The intersection of image of $(0,1) \times(0,1)$ under $f$ with $\bar{Y}$ is non-empty.

(3) For $s=0,1, f$ maps $\{s\} \times(0,1)$ into an arc that is $\delta$-equidistance from $\partial \bar{Y}$ and that is inside one of the expanding annuli in $\bar{X} \backslash \bar{Y}$.

We call $R=f([0,1] \times(0,1))$ a horizontal strip. We say a horizontal strip is maximal if it is not a proper subset of any other horizontal strips. The width $w(R)$ of a horizontal strip $R$ is defined to be the vertical length of a transverse arc to the strip, that is,

$$
w(R)=v_{q}(f(\{t\} \times(0,1))) .
$$

Vertical strips, maximal vertical strips and the width of a vertical strip are defined similarly.

Lemma 5.2 Any two maximal horizontal (respectively, vertical) strips in $\bar{Y}$ either have disjoint interiors or are identical. Furthermore, there are only finitely many distinct maximal strips, and the union of all maximal strips covers the boundary of $\bar{Y}$ except for finitely many points.

Proof If the interiors of two horizontal strips intersect, their union is also a horizontal strip. But if they are both maximal, the union cannot be larger than either of them; therefore they are identical. For a point $P$ in the boundary of $\bar{Y}$, consider the horizontal leaf passing through $P$ restricted to a $\delta$-neighborhood of $\bar{Y}$. If this horizontal arc contains no critical points of $q$, then a neighborhood of this leaf is also free of critical points, which implies that $P$ is an interior point of some horizontal strip. This proves the last assertion. To see that there are only finitely many strips, note that every maximal strip has a critical point on its boundary (otherwise one could extend it to a larger strip), and each critical point of degree $n$ can appear on the boundary of at most $n+2$ maximal strips. But the sum of the degrees of the critical points in $X$ (and therefore in $\bar{Y}$ ) is finite, and so is the number of maximal strips. The proof for vertical strips is similar.

Corollary 5.3 Let $R_{1}, \ldots, R_{p}$ be the set of maximal horizontal strips. Then the total vertical length of the boundary of $\bar{Y}$ equals twice the sum of the widths of $R_{i}$ :

$$
v_{\bar{q}}(\partial \bar{Y})=2 \sum_{i=1}^{p} w\left(R_{i}\right) .
$$

A similar statement holds for the set of maximal vertical strips. 
Example 5.4 Let $Y$ be a twice-punctured torus and and $\bar{X}$ be the following metric space. Consider a square torus and two copies of the Euclidean plane. Cut a vertical slit of the same size (say, of length $l$ ) at the middle of each of them and glue the sides of the slits as shown in Figure 2

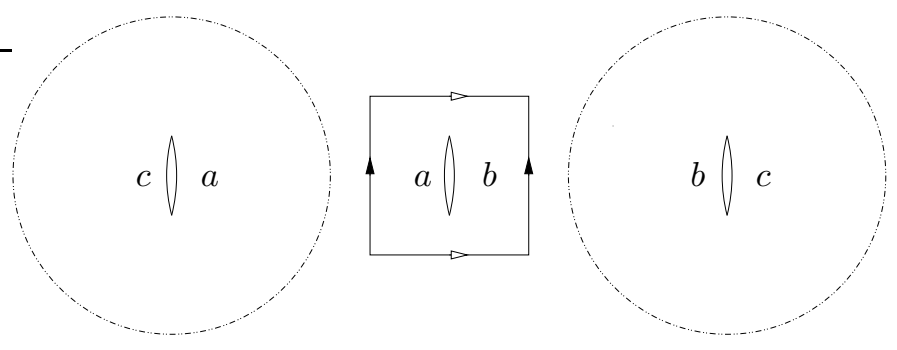

Figure 2: Space $\bar{X}$

This defines a singular Euclidean metric on $\bar{X}$ with two singular points of angle $6 \pi$ each. In this case $\bar{Y}$ is the union of the square torus with the $\operatorname{arc} c$. Let the horizontal foliation be the foliation by horizontal lines. In Figure 3 both maximal horizontal strips are shown, each with different shading. The width

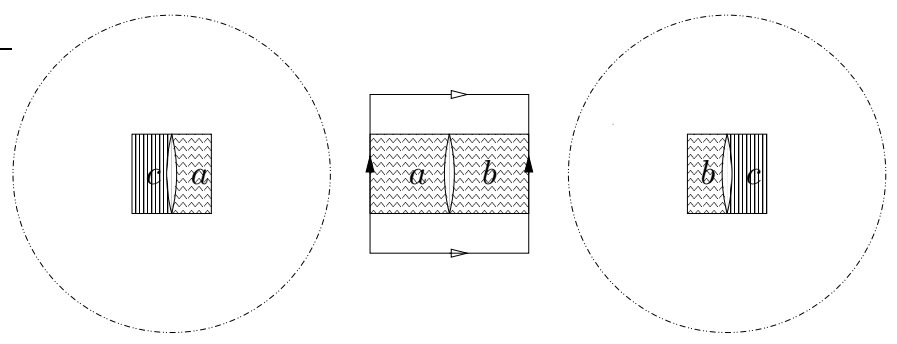

Figure 3: Horizontal strips

of each strip is $l$, so the sum of the widths of the horizontal strips is $2 l$. It is interesting that, although the boundary of $\bar{Y}$ consists of three arcs of length $l$, the total vertical length of the boundary of $\bar{Y}$ is $4 l$. This is because $\bar{Y}$ has two boundary components of length $2 l$ each, the $\operatorname{arc} c$ is traveled twice. Note that the strip intersecting arc $c$ would be degenerate if we forced the boundaries of strips to land on the boundaries of $\bar{Y}$. We set boundaries of strips to be in the expanding annuli to avoid this problem.

If $\alpha$ is a curve in $X$, a component $Y$ of $X \backslash \alpha$ is a subsurface of $X$ whose boundary consists of one or two copies of $\alpha$. We consider the annulus whose 
core curve is $\alpha$ to be a component of $X \backslash \alpha$ also. Let $\sigma$ be the hyperbolic metric on $X$.

Theorem 5.5 Let $\alpha$ be a curve in $X, \beta$ be a transverse curve to $\alpha$, and $Y$ be a component of $X \backslash \alpha$. Assume $l_{\sigma}(\beta) \leq L$. We have:

(1) If $\alpha$ is mostly vertical, then $i_{Y}\left(\beta, \nu_{+}\right) \prec D_{L}$.

(2) If $\alpha$ is mostly horizontal, then $i_{Y}\left(\beta, \nu_{-}\right) \prec D_{L}$.

Here $D_{L}$ is as in Theorem 1.3, with $\log D_{L} \asymp e^{L}$.

Proof Assume $\alpha$ is mostly vertical, that is,

$$
l_{q}(\alpha) \leq 2 v_{q}(\alpha)
$$

We prove that $i_{Y}\left(\beta, \nu_{+}\right) \leq D_{L}$. From Theorem 1.3 we have

$$
l_{q}(\beta) \leq D_{L} l_{q}(\alpha) .
$$

Let $\bar{\beta}$ be the lift of $\beta$ to $\bar{X}$, the $Y$-cover of $X$. The geometric intersection number of $\bar{\beta}$ with a horizontal leaf $h$ equals the intersection number of restrictions of $h$ and $\bar{\beta}$ to $\bar{Y}$ up to an additive error (Lemma 3.8). Let $\bar{\beta}_{0}$ be the restriction of $\bar{\beta}$ to $\bar{Y}$. Since $\left.p\right|_{\bar{Y}}$ is one-to-one, we have

$$
l_{\bar{q}}\left(\bar{\beta}_{0}\right) \leq l_{q}(\beta)
$$

and

$$
v_{q}(\alpha) \leq v_{\bar{q}}(\partial \bar{Y})
$$

Let $\left\{R_{1}, \ldots, R_{p}\right\}$ be the set of maximal horizontal strips. Now (12), (13), (14), (15) and Corollary 5.3 imply

$$
l_{\bar{q}}\left(\bar{\beta}_{0}\right) \leq 4 D_{L} \sum_{i=1}^{p} w\left(R_{i}\right) .
$$

Let $m_{i}$ be the number of times that $\bar{\beta}_{0}$ crosses $R_{i}$ and $m$ be the minimum of the $m_{i}$. Then there exists a horizontal leaf $h$ that intersects $\bar{\beta}_{0}$ at most $m$ times. That is, $i_{Y}\left(\beta, \nu_{+}\right) \leq m$. But

$$
m \sum_{i=1}^{p} w\left(R_{i}\right) \leq \sum_{i=1}^{p} m_{i} w\left(R_{i}\right) \leq l_{\bar{q}}\left(\bar{\beta}_{0}\right) .
$$

Therefore, $m \prec D_{L}$. If $\alpha$ is mostly horizontal, we can similarly show that $i_{Y}\left(\beta, \nu_{-}\right) \prec D_{L}$. 
Theorem 5.6 Let $\alpha$ be a curve in $X$ and $\beta$ be a transverse curve to $\alpha$. Assume $l_{\sigma}(\alpha) \leq \epsilon$. Then there exists a component $Y$ of $X \backslash \alpha$ such that either

(1) $Y$ is an annulus and

$$
\max \left(i_{Y}\left(\beta, \nu_{+}\right), i_{Y}\left(\beta, \nu_{-}\right)\right) \succ \frac{1}{\epsilon}, \quad \text { or }
$$

(2) $Y$ is not an annulus and

$$
\left.\log \left(\max \left(i_{Y}\left(\beta, \nu_{+}\right)\right), i_{Y}\left(\beta, \nu_{-}\right)\right)\right) \succ \frac{1}{\epsilon} .
$$

Proof Since the hyperbolic length of $\alpha$ is less than $\epsilon$, Theorem 3.7 implies that there exists a primitive annulus $A$ whose core is in the homotopy class of $\alpha$ and whose modulus is $m \asymp \frac{1}{\epsilon}$. Let $d$ be the distance between the boundary components of $A$, and let $\gamma_{0}$ be the inner boundary of $A$. If $A$ is flat, let $Y$ be $A$ and $\mathrm{k}=\mathrm{m}$; if $A$ is expanding, let $Y$ be the component of $X \backslash \alpha$ that contains $A$ and let

$$
k=\frac{d}{l_{q}\left(\gamma_{0}\right)} .
$$

Lemma [3.6 implies that $\log k \asymp m$.

Let $\bar{A}, \bar{\alpha}$ and $\bar{\beta}$ be the lifts of $A, \alpha_{q}$ and $\beta_{q}$ to $\bar{X}$, respectively, and let $\bar{\beta}_{0}$ be the restriction of $\bar{\beta}$ to $\bar{Y}$. The arc $\bar{\beta}_{0}$ has to cross $\bar{A}$; therefore, $l_{\bar{q}}\left(\bar{\beta}_{0}\right) \geq d$. Also, $\bar{\alpha}$ is a geodesic; therefore, $l_{\bar{q}}(\bar{\alpha}) \leq l_{q}\left(\gamma_{0}\right)$. We have

$$
\frac{l_{\bar{q}}\left(\bar{\beta}_{0}\right)}{l_{\bar{q}}(\bar{\alpha})} \succ k \text {. }
$$

The $\operatorname{arc} \bar{\beta}_{0}$ is either mostly vertical or mostly horizontal. That is,

$$
2 v_{\bar{q}}\left(\bar{\beta}_{0}\right) \geq l_{\bar{q}}\left(\bar{\beta}_{0}\right) \quad \text { or } \quad 2 h_{\bar{q}}\left(\bar{\beta}_{0}\right) \geq l_{\bar{q}}\left(\bar{\beta}_{0}\right) .
$$

Assume the first inequality holds. Let $\left\{R_{1}, \ldots, R_{p}\right\}$ be the set of horizontal strips. Corollary [5.3 (18) and the pigeonhole principle imply that $\bar{\beta}_{0}$ has to intersect some $R_{i}$ at least (up to a multiplicative constant) $k$ times. But the geometric intersection number between $\bar{\beta}$ and the horizontal foliation is equal to the intersection number of $\bar{\beta}_{0}$ with a horizontal leaf up to an additive constant (Lemma 3.8). That is,

$$
i_{Y}\left(\beta, \nu_{+}\right) \succ k \text {. }
$$

In case the second inequality in (19) holds, we consider vertical strips and we show similarly that $\bar{\beta}_{0}$ intersects a vertical leaf at least (up to a multiplicative constant) $k$ times. That is

$$
i_{Y}\left(\beta, \nu_{-}\right) \succ k .
$$

This finishes the proof of the theorem. 


\section{Proof of the main theorem}

The main theorem is a corollary of Theorems 5.5 and 5.6. First we recall a few facts about the Teichmüller space. The Teichmüller space of $S$ is the space of conformal structures on $S$, where two structures are considered to be equivalent if there is a conformal map between them isotopic to the identity. There are several natural metrics defined on $\mathcal{T}(S)$, all inducing the same natural topology. We work with the Teichmüller metric, which assigns to $X_{1}, X_{2} \in \mathcal{T}(S)$ the distance

$$
d_{\mathcal{T}(S)}\left(X_{1}, X_{2}\right)=\frac{1}{2} \log (k),
$$

where $k$ is the smallest dilatation of a quasi-conformal homeomorphism from $X_{1}$ to $X_{2}$ that is isotopic to the identity.

Geodesics in the Teichmüller space of $S$ are determined by the quadratic differentials. Let $q$ be a quadratic differential on a Riemann surface $X \in \mathcal{T}(S)$. Define $g(0)=X$ and, for $t \in \mathbb{R}, g(t)$ to be the conformal structure obtained by scaling the horizontal foliation of $q$ by a factor of $e^{t}$, and the vertical by a factor of $e^{-t}$. Then $g: \mathbb{R} \rightarrow \mathcal{T}(S)$ is a geodesic in $\mathcal{T}(S)$ parametrized by arc length. The corresponding family of quadratic differentials is denoted by $q_{t}$. For a curve $\alpha$ in $S$, the horizontal and vertical lengths of $\alpha$ vary with time as follows:

$$
h_{q_{t}}(\alpha)=h_{q}(\alpha) e^{-t} \quad \text { and } \quad v_{q_{t}}(\alpha)=v_{q}(\alpha) e^{t} .
$$

We define a constant $K_{\alpha}$, which measures the relative complexity of $\mu^{+}$and $\mu^{-}$from the point of view of $\alpha$, as follows: For each subsurface $Y$ of $X$,

(1) If $Y$ is not an annulus, define $K_{Y}=\log K$ where $K$ is the smallest positive number such that $\mu_{Y}^{+}$and $\mu_{Y}^{-}$have a common $K$-quasi-parallel.

(2) If $Y$ is an annulus, define $K_{Y}=i_{Y}\left(\mu^{+}, \mu^{-}\right)$.

Now, define $K_{\alpha}$ to be the largest $K_{Y}$ where $Y$ is a component of $X \backslash \alpha$.

Theorem 6.1 Let $\left(g, \mu^{+}, \mu^{-}\right)$be a geodesic in $\mathcal{T}(S)$ and $\alpha$ be a simple closed curve in $S$. Assume $\alpha$ is balanced at $t_{0}$. Then

$$
\frac{1}{l_{g}(\alpha)} \asymp \frac{1}{l_{g\left(t_{0}\right)}(\alpha)} \asymp K_{\alpha} .
$$

Proof By definition of $l_{g}(\alpha)$ we have

$$
\frac{1}{l_{g}(\alpha)} \succ \frac{1}{l_{g\left(t_{0}\right)}(\alpha)}
$$

Theorem 5.6 implies that for every time $t$ there exists a component $Y$ of $X \backslash \alpha$ such that either 
(1) $Y$ is an annulus and $i\left(\mu^{+}, \mu^{-}\right) \succ 1 / l_{g(t)}(\alpha) 6$ or

(2) $Y$ is not an annulus and $\log \left(K_{Y}\right) \succ 1 / l_{g(t)}(\alpha)$.

Therefore,

$$
K_{\alpha} \succ \frac{1}{l_{g}(\alpha)} .
$$

Let $\epsilon=l_{g\left(t_{0}\right)}(\alpha)$. It remains to show that, for every component $Y$ of $X \backslash \alpha$, $\frac{1}{\epsilon} \succ K_{Y}$.

If $Y$ is not an annulus, pick a transverse curve $\beta$ of length less than $L$, where $e^{L} \asymp \frac{1}{\epsilon}$. Since $\alpha$ is mostly horizontal and mostly vertical simultaneously, Theorem [5.6 implies that $\beta_{Y}$ (see Section 2 for definition) is $D_{L}$-quasi-parallel to $\mu_{Y}^{+}$and $\mu_{Y}^{-}$. Therefore, $K_{Y} \prec e^{L} \asymp \frac{1}{\epsilon}$.

If $Y$ is an annulus, then $\bar{Y}$ is a flat annulus. Let $d$ be the distance between the boundary components of $\bar{Y}$ and $l$ be the length of one of the boundary components of $\bar{Y}$. We know that

$$
\frac{d}{l}=\operatorname{Mod}(\bar{Y}) \prec \frac{1}{\epsilon} .
$$

Visualizing $\bar{Y}$ standing vertically, the restrictions of $\mu^{+}$and $\mu^{-}$to $\bar{Y}$ have angles $\frac{\pi}{2}$ and $-\frac{\pi}{2}$ respectively (because $\alpha$ is balanced). Therefore, an arc in $\mu_{Y}^{+}$intersects an arc in $\mu_{Y}^{-}$(up to an additive error) $\frac{d}{l}$ times. This implies (Lemma 3.8)

$$
K_{Y}=i_{Y}\left(\mu^{+}, \mu^{-}\right) \prec \frac{1}{\epsilon} .
$$

Remark 6.2 The above theorem is silent with respect to the case when $\alpha$ is never balanced (i.e., if the horizontal or the vertical length of $\alpha$ is zero along $g)$. It is easy to see that, if $\alpha$ is homotopic to a closed leaf of $\mu^{+}$or $\mu^{-}$, then $l_{g}(\alpha)=0$, and if not, then $l_{g}(\alpha)$ is positive (consider, for example, the geodesic corresponding to the quadratic differential in Example 5.4). However, one cannot approximate the value of $l_{g}(\alpha)$ using the underlying foliations only.

\section{Subsurface distances}

In this section we recall some of the properties of the subsurface distances from [7] and [8] and prove Theorems 1.5, 1.6] and 1.7

\footnotetext{
${ }^{6}$ One can always find a curve $\beta$ such that $i\left(\mu^{+}, \mu^{-}\right) \geq \max \left(i_{Y}\left(\beta, \mu^{+}\right), i_{Y}\left(\beta, \mu^{-}\right)\right)$.
} 
First we define a metric on $\mathcal{C} S(S)$ as follows. For curve systems $\Gamma$ and $\Gamma^{\prime}$ in $\mathcal{C} S(S)$, define $d_{S}\left(\Gamma, \Gamma^{\prime}\right)$ to be equal to one if $[\Gamma] \neq\left[\Gamma^{\prime}\right]$ and if an element of $[\Gamma]$ and an element of $\left[\Gamma^{\prime}\right]$ have representatives that are disjoint from each other. Let the metric on $\mathcal{C} S(S)$ be the maximal metric having the above property. That is, $d_{S}\left(\Gamma, \Gamma^{\prime}\right)=n$ if $\Gamma=\Gamma_{0}, \Gamma_{1}, \ldots, \Gamma_{n}=\Gamma^{\prime}$ is the shortest sequence of curve systems on $S$ such that $\Gamma_{i-1}$ is distance one from $\Gamma_{i}, i=1, \ldots, n$.

Theorem 7.1 (Masur, Minsky) Let $\Gamma$ and $\Gamma^{\prime}$ be curve systems in $S$. Then

$$
d_{S}\left(\Gamma, \Gamma^{\prime}\right) \prec \log \left(i_{S}\left(\Gamma, \Gamma^{\prime}\right)\right)
$$

Remark 7.2 The theorem is true for any subsurface of $S$ that is not an annulus. If $Y$ is an annulus then

$$
d_{Y}\left(\Gamma, \Gamma^{\prime}\right)=i_{Y}\left(\Gamma, \Gamma^{\prime}\right)+1
$$

Conversely, a bound on all subsurface distances gives a bound on the intersection number between two curves.

Theorem 7.3 (Masur, Minsky) For every $D>0$, there exists $K>0$ such that, for $\Gamma, \Gamma^{\prime} \in \mathcal{C}(S)$, if $i_{S}\left(\Gamma, \Gamma^{\prime}\right)>K$, then there exists a subsurface $Y$ of $S$ such that

$$
d_{Y}\left(\Gamma, \Gamma^{\prime}\right)>D
$$

See [7] and [8] for the proofs of the above theorems and detailed discussion.

Let $\mu^{+}$and $\mu^{-}$be foliations (projectivized measured foliations or unmeasured foliations) on $S$. Define $d_{Y}\left(\mu^{+}, \mu^{-}\right)$to be the distance in $\mathcal{C} S(Y)$ between the restrictions of $\mu^{+}$and $\mu^{-}$to $Y$ (see Section 2 for definition). We call this the $Y$-distance between $\mu^{+}$and $\mu^{-}$.

As we mentioned in the introduction, Minsky has shown in 13 that every short curve of a hyperbolic 3-manifold $\left(N, \mu^{+}, \mu^{-}\right)$homeomorphic to $S \times \mathbb{R}$ appears as a boundary component of a subsurface $\mathrm{Y}$ of $S$ where the $Y$-distance of $\mu^{+}$and $\mu^{-}$is large. Now we are ready to prove Theorems 1.5 and 1.6. For simplicity, we present proofs without keeping track of constants.

Proof of Theorem 1.5] The hyperbolic 3-manifold $\left(N, \mu^{+}, \mu^{-}\right)$has bounded geometry if and only if there a uniform bound on $d_{Y}\left(\mu^{+}, \mu^{-}\right)$, for every subsurface $Y$ of $S$. Also, our main theorem (Theorem 6.1) implies that a geodesic $\left(g, \mu^{+}, \mu^{-}\right)$has bounded geometry if there exists a uniform bound on $K_{\alpha}$, for every curve $\alpha$ in $S$. We have to show these two conditions are equivalent. 
Let $Z$ be a subsurface, $\alpha$ be a boundary component of $Z$ and $Y$ be a component of $S \backslash \alpha$ containing $Z$. A bound on $K_{\alpha}$ provides a bound on $i_{Y}\left(\mu^{+}, \beta\right)$ and $i_{Y}\left(\beta, \mu^{-}\right)$, where $\beta$ is an arc in $Y$. This provides a bound for $i_{Z}\left(\mu^{+}, \beta\right)$ and $i_{Z}\left(\beta, \mu^{-}\right)$, which in turn bound $d_{Z}\left(\mu^{+}, \mu^{-}\right)$(Theorem 7.1). Therefore a uniform bound for $K_{\alpha}$ provides a uniform bound for $d_{Z}\left(\mu^{+}, \mu^{-}\right)$.

On the other hand, a bound on all subsurface distances gives a bound for every $i_{Y}\left(\mu^{+}, \mu^{-}\right)$(Theorem [7.3). Therefore, the two conditions are equivalent.

Proof of Theorem 1.6 If a curve $\alpha$ is short in $\left(N, \mu^{+}, \mu^{-}\right)$, then it is a boundary of a subsurface $Z$, where $d_{Z}\left(\mu^{+}, \mu^{-}\right)$is large. As we saw above, $K_{\alpha}\left(\mu^{+}, \mu^{-}\right)$has to be large (small $K_{\alpha}$ implies small $d_{Z}\left(\mu^{+}, \mu^{-}\right)$). Therefore, $l_{g}(\alpha)$ has to be small as well.

Here we provide a family of examples satisfying Theorem 1.7

Theorem 1.7 There exist a sequence of manifolds $\left(N_{n}, \mu_{n}^{+}, \mu_{n}^{-}\right)$, corresponding Teichmüller geodesics $\left(g_{n}, \mu_{n}^{+}, \mu_{n}^{-}\right)$and a curve $\gamma$ in $S$ such that $l_{N_{n}}(\gamma) \geq$ $c>0$ for all $n$, but

$$
l_{g_{n}}(\gamma) \rightarrow 0 \quad \text { as } \quad n \rightarrow \infty
$$

Proof Let $\left(g, \mu^{+}, \mu^{-}\right)$be a geodesic in $\mathcal{T}(S)$, and let $\phi_{1}$ (respectively, $\phi_{2}$ ) be a mapping class of $S$ whose support is a subsurface $Y_{1}\left(Y_{2}\right)$ and whose restriction to $Y_{1}\left(Y_{2}\right)$ is pseudo-Anosov. Assume that $Y_{1}$ and $Y_{2}$ intersect each other nontrivially (that is, the boundary components of one have nontrivial projections to the other), and that they fill a subsurface $Z$ which is a component of $S \backslash \alpha$, for some curve $\alpha$ (in fact, it would be enough to assume $Z$ is a proper subsurface of $S$ ). Define

$$
\mu_{n}^{+}=\phi_{1}^{n}\left(\mu^{+}\right) \quad \text { and } \quad \mu_{n}^{-}=\phi_{2}^{n}\left(\mu^{-}\right) .
$$

Consider the sequence of geodesics $\left(g_{n}, \mu_{n}^{+}, \mu_{n}^{-}\right)$and the corresponding sequence of hyperbolic 3-manifolds $\left(N_{n}, \mu_{n}^{+}, \mu_{n}^{-}\right)$. Let $K_{n}$ be the smallest number such that the restrictions of $\mu_{n}^{+}$and $\mu_{n}^{-}$to $Z$ have a common $K_{n}$-quasi-parallel. To prove the theorem, we show that the length of $\alpha$ remains bounded in $N_{n}$, but goes to zero in $g_{n}$, as $n$ approaches infinity. It is enough to show that, as $n$ approaches infinity,

(1) $d_{\bar{Z}}\left(\mu_{n}^{+}, \mu_{n}^{-}\right)$stays bounded, for any subsurface $\bar{Z}$ which includes $\alpha$ as a boundary component, and 
(2) $K_{n}$ approaches infinity.

The curve $\alpha$ is outside of $Y_{1}$ and $Y_{2}$. Therefore, each of $Y_{1}$ and $Y_{2}$ either is disjoint from $\bar{Z}$ or has a boundary component with has a nontrivial projection to $\bar{Z}\left(\bar{Z} \subset Y_{1}\right.$ is excluded). Assume the restrictions of both $\partial Y_{1}$ and $\partial Y_{2}$ to $\bar{Z}$ are nontrivial. Since the supports of $\phi_{1}$ and $\phi_{2}$ are $Y_{1}$ and $Y_{2}$, respectively, we have

$$
i_{\bar{Z}}\left(\mu_{n}^{+}, \partial Y_{1}\right)=i_{Z}\left(\mu^{+}, \partial Y_{1}\right) \quad \text { and } \quad i_{\bar{Z}}\left(\mu_{n}^{-}, \partial Y_{2}\right)=i_{\bar{Z}}\left(\mu^{-}, \partial Y_{2}\right)
$$

Therefore,

$$
\begin{aligned}
d_{\bar{Z}}\left(\mu_{n}^{+}, \mu_{n}^{-}\right) & \leq d_{\bar{Z}}\left(\mu_{n}^{+}, \partial Y_{1}\right)+d_{\bar{Z}}\left(\partial Y_{1}, \partial Y_{2}\right)+d_{\bar{Z}}\left(\partial Y_{2}, \mu_{n}^{-}\right) \\
& \prec \log i_{\bar{Z}}\left(\mu_{n}^{+}, \partial Y_{1}\right)+d_{\bar{Z}}\left(\partial Y_{1}, \partial Y_{2}\right)+\log i_{\bar{Z}}\left(\partial Y_{2}, \mu_{n}^{-}\right) \\
& =\log i_{\bar{Z}}\left(\mu^{+}, \partial Y_{1}\right)+d_{\bar{Z}}\left(\partial Y_{1}, \partial Y_{2}\right)+\log i_{\bar{Z}}\left(\partial Y_{2}, \mu^{-}\right)
\end{aligned}
$$

which is independent of $n$. If only one of them, say $Y_{1}$, is disjoint from $\bar{Z}$, then

$$
i_{\bar{Z}}\left(\mu_{n}^{+}, \partial Y_{2}\right)=i_{\bar{Z}}\left(\mu^{+}, \partial Y_{2}\right) \text {. }
$$

Now we can write

$$
d_{\bar{Z}}\left(\mu_{n}^{+}, \mu_{n}^{-}\right) \leq d_{\bar{Z}}\left(\mu_{n}^{+}, \partial Y_{2}\right)+d_{\bar{Z}}\left(\partial Y_{2}, \mu_{n}^{-}\right)
$$

and argue as before. And finally, if both $Y_{1}$ and $Y_{2}$ are disjoint from $\bar{Z}$, then

$$
i_{\bar{Z}}\left(\mu_{n}^{+}, \mu_{n}^{-}\right)=i_{\bar{Z}}\left(\mu^{+}, \mu^{-}\right) .
$$

This finishes the proof of part (1).

Let $\omega_{n}$ be the arc that is $K_{n}$-quasi-parallel to $\mu_{n}^{+}$and $\mu_{n}^{-}$. Since $Y_{1}$ and $Y_{2}$ fill $Z, \omega_{n}$ has to intersect one of them nontrivially. By taking a subsequence, we can assume that $\omega_{n}$ intersects $Y_{1}$ nontrivially (the proof for the subsequence intersecting $Y_{2}$ nontrivially is similar). We have

$$
\begin{aligned}
d_{Y_{1}}\left(\mu_{n}^{+}, \mu_{n}^{-}\right) & \leq d_{Y_{1}}\left(\mu_{n}^{+}, \omega_{n}\right)+d_{Y_{1}}\left(\omega_{n}, \mu_{n}^{-}\right) \\
& \prec 2 \log K_{n} .
\end{aligned}
$$

Since $\phi_{1}$ restricted to $Y_{1}$ is pseudo-Anosov, we have (see [7])

$$
d_{Y_{1}}\left(\mu_{n}^{+}, \partial Y_{2}\right) \rightarrow \infty \quad \text { as } n \rightarrow \infty \text {. }
$$

But

$$
\begin{aligned}
d_{Y_{1}}\left(\mu_{n}^{-}, \partial Y_{2}\right) & \prec \log i_{Y_{1}}\left(\mu_{n}^{-}, \partial Y_{2}\right) \\
& \leq \log i_{Z}\left(\mu_{n}^{-}, \partial Y_{2}\right) \\
& =\log i_{Z}\left(\mu^{-}, \partial Y_{2}\right),
\end{aligned}
$$

which is bounded. Therefore,

$$
d_{Y_{1}}\left(\mu_{n}^{+}, \mu_{n}^{-}\right) \rightarrow \infty \quad \text { as } \quad n \rightarrow \infty .
$$

This implies that $K_{n}$ approaches infinity as $n$ goes to infinity, which proves part (2). 


\section{References}

[1] L Bers, Simultaneous uniformization, Bull. Amer. math. Soc. 66 (1960) 94-97 MathReview

[2] F Bonahon, Bouts des variétés hyperboliques de dimension 3, Ann. Math. 124 (1986) 71-158 MathReview

[3] J Brock, D Canary, Y Minsky, The classification of Kleinian surface groups II: the ending lamination conjecture, in preparation

[4] F P Gardiner, Quasiconformal Teichmüller theory, Mathematical Surveys and Monographs 76, Amer. Math. Soc. (2000) MathReview

[5] F P Gardiner, H Masur, Extremal length geometry of Teichmüller space, Complex Variables Theory Appl. 16 (2000) 209-237 MathReview

[6] B Maskit, Comparison of hyperbolic and extremal lengths, Ann. Acad. Sci. Fenn. Ser. A I Math. 10 (1985) 381-386 MathReview

[7] H Masur, Y Minsky, Geometry of the complex of curves I, hyperbolicity, Invent. Math. 138 (1999) 103-149 MathReview

[8] H Masur, Y Minsky, Geometry of the complex of curves II, hierarchical structure, Geom. and Func. Anal. 10 (2000) 902-974 MathReview

[9] Y Minsky, Harmonic maps, length, and energy in Teichmüller space, J. Diff. Geo. 35 (1992) 151-217 MathReview

[10] Y Minsky, Teichmüller geodesics and ends of hyperbolic 3-manifolds, Topology 32 (1993) 625-647 MathReview

[11] Y Minsky The classification of punctured-torus group, Annals of Math. 149 (1999) 559-626 MathReview

[12] Y Minsky Kleinian groups and the complex of curves, Geom. Topol. 4 (2000) 117-148

[13] Y Minsky The classification of Kleinian surface groups I: models and bounds, arXiv:math.GT/0302208

[14] Y Minsky Bounded geometry for Kleinian groups, Invent. Math. 146 (2001) 143-192 MathReview

[15] K Rafi, Hyperbolic 3-manifolds and geodesics in Teichmüller space, $\mathrm{PhD}$ thesis, SUNY at Stony Brook (August 2001)

[16] M Rees, The geometric model and large Lipschitz equivalence direct from Teichmüller geodesic, preprint, http://www.liv.ac.uk/ maryrees/papershomepage.html

[17] K Strebel, Quadratic differentials, Ergebnisse series 3, Springer-Verlag (1984) MathReview

[18] W Thurston, The Geometry and Topology of 3-Manifolds, Princeton University Lecture Notes (1982) http://www.msri.org/publications/books/gt3m/ 